\title{
WestVirginiaUniversity
}

THE RESEARCH REPOSITORY @ WVU

Graduate Theses, Dissertations, and Problem Reports

2005

\section{On the s-Hamiltonian index of a graph}

Yehong Shao

West Virginia University

Follow this and additional works at: https://researchrepository.wvu.edu/etd

\section{Recommended Citation}

Shao, Yehong, "On the s-Hamiltonian index of a graph" (2005). Graduate Theses, Dissertations, and Problem Reports. 1646.

https://researchrepository.wvu.edu/etd/1646

This Thesis is protected by copyright and/or related rights. It has been brought to you by the The Research Repository @ WVU with permission from the rights-holder(s). You are free to use this Thesis in any way that is permitted by the copyright and related rights legislation that applies to your use. For other uses you must obtain permission from the rights-holder(s) directly, unless additional rights are indicated by a Creative Commons license in the record and/ or on the work itself. This Thesis has been accepted for inclusion in WVU Graduate Theses, Dissertations, and Problem Reports collection by an authorized administrator of The Research Repository @ WVU. For more information, please contact researchrepository@mail.wvu.edu. 


\title{
On the $s$-hamiltonian Index of a Graph
}

\author{
Yehong Shao \\ Thesis submitted to the \\ College of Engineering and Mineral Resources \\ at West Virginia University \\ in partial fulfillment of the requirements \\ for the degree of \\ Master of Science \\ in \\ Computer Science
}

Elaine M. Eschen, Ph.D, Chair

Hong-Jian Lai, Ph.D.

Frances L. VanScoy, Ph.D.

Lane Department of Computer Science and Engineering

Morgantown, West Virginia

2005

Keywords: line graph, hamiltonian index, hamiltonicity, contraction

Copyright 2005 Yehong Shao 


\section{ABSTRACT \\ On the $s$-hamiltonian Index of a Graph}

\section{Yehong Shao}

In modeling communication networks by graphs, the problem of designing $s$-faulttolerant networks becomes the search for $s$-hamiltonian graphs. This thesis is a study of the $s$-hamiltonian index of a graph $G$.

A path $P$ of $G$ is called an arc in $G$ if all the internal vertices of $P$ are divalent vertices of $G$. We define $l(G)=\max \{m: G$ has an arc of length $m$ that is not both of length 2 and in a $\left.K_{3}\right\}$. We show that if a connected graph $G$ is not a path, a cycle or $K_{1,3}$, then for a given $s$, we give the best known bound of the $s$-hamiltonian index of the graph. 


\section{Acknowledgements}

I sincerely express my appreciation to my thesis advisor, Dr. Elaine M. Eschen, for

her guidance, advice, and continual encouragement. It is a pleasure to work under her supervision.

I would also like to thank my other committee members: Dr. Hong-Jian Lai, and Dr. Frances L. Van Scoy, for their help during my studies. 


\section{DEDICATION}

To

my father Jinfu, my mother Luolan

$$
\text { and }
$$

my sister Honglian 


\section{Contents}

1 Introduction 1

1.1 Background . . . . . . . . . . . . . . . . . 1

1.2 Definitions . . . . . . . . . . . . . . . . . . . 3

$2 \quad s$-Hamilton Index $\quad 4$

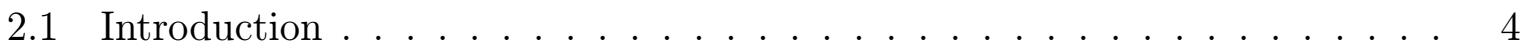

2.2 Proof of the Main Theorem . . . . . . . . . . . . . . . 5 


\section{Chapter 1}

\section{Introduction}

\section{$1.1 \quad$ Background}

Multiprocessors are widely used in recent years. Fault-tolerant multiprocessors are particularly useful in massive parallel systems. How to communicate among the processes running in parallel on the multiple processors becomes an important problem. The network that connects multiprocessors is called an interconnection network.

To design a reliable interconnection network, one expects that the network is faulttolerant. There are two types of failures in a multiprocessor system, processor failure and link failure. It is important that the computer system still works when one or more processors fail.

We use a graph as a theoretic model to represent an underlying interconnection network. Terminology and notations not defined here can be found in [1]. Let $G=(V, E)$ be a graph. The vertex set $V(G)$ represents the set of processors and the edge set $E(G)$ represents the set of links between processors. A processor failure corresponds to the deletion of a vertex from a graph.

The topology of a network is the way the nodes and links are connected. Figure 1.1 
shows two different topologies.

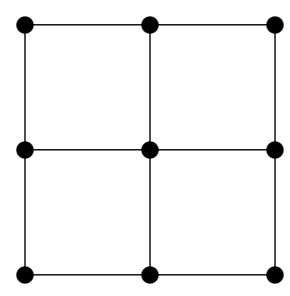

(a) a mesh topology

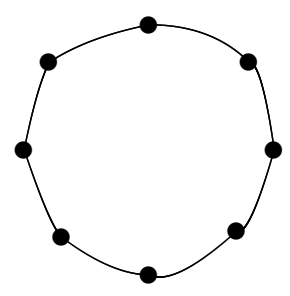

(b) a ring topology

Figure 1.1: Example networks

In many network designs, it is desirable to maintain a fixed network topology. Reliability consideration expects that the network possesses the capability that after a small number of processor failures, the network can reconfigure to keep the same network topology.

A graph $G$ is hamiltonian if there exists a cycle containing all the vertices of $G$.

One of the common network topologies uses a ring connection joining all the processors (see Figure 1.1(b)), which corresponds to a hamiltonian cycle of the graph $G$ modeling such a network.

A network is a $k$-fault-tolerant network for a ring if for any $k$-processor-failure, the resulting network contains a ring including all of the non-faulty processors. This motivates the following definition.

A graph $G$ is called $s$-hamiltonian, if the removal of any $k$ vertices, $0 \leq k \leq s \leq$ $|V(G)|$, results in a hamiltonian graph. 


\subsection{Definitions}

We consider finite simple connected graphs only. For a graph $G$ and a vertex $v \in V(G)$, define

$$
N_{G}(v)=\{u \in V(G): u \text { is adjacent to } v \text { in } G\}
$$

and

$$
E_{G}(v)=\{e \in E(G): e \text { is incident with } v \text { in } G\} .
$$

The line graph of a graph $G$, denoted by $L(G)$ or $L^{1}(G)$, has $E(G)$ as its vertex set, where two vertices in $L(G)$ are adjacent if and only if the corresponding edges in $G$ have a common vertex. Notice that the vertex set in a line graph $L(G)$ corresponding to each $E_{G}(v)$ in $G$ induces a complete graph. Denote each complete graph in $L(G)$ which corresponds to $E_{G}(v)$ in $G$ by $K_{v}$. Then $\left\{E\left(K_{v}\right): v \in V(G)\right\}$ is an edge partition of $L(G)$ and we say this is a complete partition of $L(G)$. For an integer $m \geq 1$, we define $L^{m}(G)=L\left(L^{m-1}(G)\right)$ with $L^{0}(G)=G$.

In 1973, Chartrand [2] introduced the hamiltonian index of a connected graph $G$ that is not a path to be the minimum number of applications of the line graph operator so that the resulting graph is hamiltonian. He showed that the hamiltonian index exists as a finite number. In 1983, Clark and Wormald [3] extended this idea of Chartrand and introduced the hamiltonian-like indices. Here we define the $s$-hamiltonian index.

Let $s \geq 0$ be an integer. The $s$-hamiltonian index, $h_{s}(G)$, of a connected graph $G$ is the least nonnegative integer $m$ such that $L^{m}(G)$ is $s$-hamiltonian. Note that when $s=0$, a 0 -hamiltonian graph is a hamiltonian graph and $h_{0}(G)=h(G)$ is the Hamilton index of a graph $G$. 


\section{Chapter 2}

\section{$s$-Hamilton Index}

\section{$2.1 \quad$ Introduction}

In this chapter we prove our main result, stated in Theorem 2.1.1. This provides the best known bound for the $s$-hamiltonian index of a graph.

A nontrivial path $P$ of $G$ is called an $\operatorname{arc}$ in $G$ if all the internal vertices of $P$ are divalent vertices of $G$. We define $l(G)=\max \{m: G$ has an arc of length $m$ that is not both of length 2 and in a $\left.K_{3}\right\}$. Note that $l(G) \geq 1$.

Theorem 2.1.1 Let $G$ be a simple connected graph that is not a path, a cycle, or $K_{1,3}$ with $l(G)=l$. Then $h_{s}(G) \leq l+s+1$.

In the case that $s=0$ Theorem 2.1.1 yields the theorem below, extends a former result by Lai.

Theorem 2.1.2 (Lai, [5]) Let $G$ be a simple connected graph that is not a path, a cycle, or $K_{1,3}$ with $l(G)=l$. Then $h(G) \leq l+1$. 


\subsection{Proof of the Main Theorem}

Let $O(G)$ denote the set of all vertices in $G$ with odd degree. A graph $G$ is Eulerian if both $O(G)=\emptyset$ and $G$ is connected. A spanning closed trail of $G$ is called a spanning Eulerian subgraph of $G$. A subgraph $H$ of $G$ is dominating if $G-V(H)$ is edgeless. If a closed trail $C$ of $G$ satisfies $E(G-V(C))=\emptyset$, then $C$ is called a dominating Eulerian subgraph.

Theorem 2.2.1 reveals the relationship between a dominating Eulerian subgraph in $H$ and a hamiltonian cycle in $L(H)$.

Theorem 2.2.1 (Harary and Nash-Williams, [4]) Let $H$ be a graph with $|E(H)| \geq 3$. The line graph $L(H)$ of a graph $H$ is hamiltonian if and only if $H$ has a dominating Eulerian subgraph.

An edge cut $X$ of $G$ is essential if each side of $G-X$ has an edge.

For a graph $G$ and a subset $X \subseteq E(G)$, the contraction $G / X$ is the graph obtained from $G$ by identifying the two end vertices of each edge in $X$ and then deleting the edges in $X$. Note that loops and/or multiple edges may result from a contraction.

Lemma 2.2.2 Let $G$ be a connected graph and $H$ an edge subset of $G$.

(i) if $H$ is an edge set consisting of loops of $G$ and $G / H$ has a spanning Eulerian subgraph, then $G$ has a spanning Eulerian subgraph;

(ii) if $H$ is a pair of parallel edges or the edge set of a $C_{3}$ and $G / H$ has a spanning Eulerian subgraph, then $G$ has a spanning Eulerian subgraph.

Proof (i) Let $T$ be a spanning Eulerian subgraph of $G / H$. Then $T$ or $T+H$ is a spanning Eulerian subgraph of $G$.

(ii) Let $T$ be a spanning Eulerian subgraph of $G / H$. 
Case $1 H=\left\{e_{1}, e_{2}\right\}$ is an edge set of parallel edges of $G$ (see Case 1 in Figure 2.1). Let $v_{1}, v_{2}$ be the two endpoints vertices of the edges in $H$ and $v_{H}$ the vertex in $G / H$ onto which $H$ is contracted. Let $T^{\prime}$ be the graph obtained from $T-v_{H}$ by adding vertices $v_{1}$ and $v_{2}$ with $N_{T^{\prime}}\left(v_{1}\right)=N_{G}\left(v_{1}\right) \cap N_{T}\left(v_{H}\right)$ and $N_{T^{\prime}}\left(v_{2}\right)=N_{G}\left(v_{2}\right) \cap N_{T}\left(v_{H}\right)$. Since $d_{T}\left(v_{H}\right)$ is even, $d_{T^{\prime}}\left(v_{1}\right)+d_{T^{\prime}}\left(v_{2}\right)$ is even. If $d_{T^{\prime}}\left(v_{1}\right)$ and $d_{T^{\prime}}\left(v_{2}\right)$ are both even, then $T^{\prime}+\left\{e_{1}, e_{2}\right\}$ is a spanning Eulerian subgraph of $G$; if $d_{T^{\prime}}\left(v_{1}\right)$ and $d_{T^{\prime}}\left(v_{2}\right)$ are both odd, then $T^{\prime}+e_{1}$ is a spanning Eulerian subgraph of $G$.

Case $2 H=\left\{e_{1}, e_{2}, e_{3}\right\}$ is the edge set of a $C_{3}$ in $G$. Let $v_{1}, v_{2}, v_{3}$ be the three endpoints of the edges in $H$ (see Case 2 in Figure 2.1) and $v_{H}$ the vertex in $G / H$ onto which $H$ is contracted. Let $T^{\prime}$ be defined as in Case 7. Since $d_{T}\left(v_{H}\right)$ is even, $d_{T^{\prime}}\left(v_{1}\right)+d_{T^{\prime}}\left(v_{2}\right)+d_{T^{\prime}}\left(v_{3}\right)$ is even. If $d_{T^{\prime}}\left(v_{1}\right), d_{T^{\prime}}\left(v_{2}\right)$ and $d_{T^{\prime}}\left(v_{3}\right)$ are all even, then $T+H$ is a spanning Eulerian subgraph of $G$; if two of them are odd and we assume without loss of generality that $d_{T^{\prime}}\left(v_{1}\right)$ and $d_{T^{\prime}}\left(v_{2}\right)$ are odd, then $T+\left\{e_{1}, e_{2}\right\}$ is a spanning Eulerian subgraph of $G$.

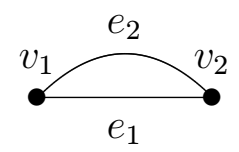

Case 1

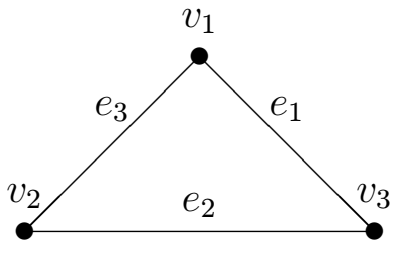

Case 2

Figure 2.1: The edge set $H$ of Lemma 2.2.2

Lemma 2.2.3 Let $G$ be a connected graph without essential edges cuts of size 1 and $G_{1}$ the graph obtained by contracting all the triangles and multiple edges repeatedly from $G$. If $\left|V\left(G_{1}\right)\right| \leq 4$, then $G$ has a dominating Eulerian subgraph.

Proof By the assumptions, $G_{1}$ is simple, connected and has no 3-cycles. 


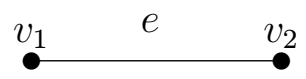

Figure 2.2.1

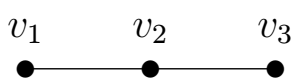

Figure 2.2.2

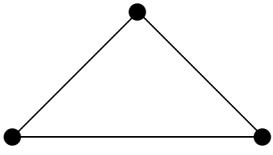

Figure 2.2.3

Figure 2.2: $G_{1}$

Case $1\left|V\left(G_{1}\right)\right|=1$. By Lemma 2.2.2, G has a spanning Eulerian subgraph.

Case $2\left|V\left(G_{1}\right)\right|=2$ (See Figure 2.2.1). Since $G$ has no essential edge cuts of size 1, one of the vertices is a vertex of $G$. We assume that it is $v_{1}$. We delete $v_{1}$ and the resulting graph is $K_{1}$. By Lemma 2.2.2, G-v has a spanning Eulerian subgraph $T$, and so $T$ is a dominating Eulerian subgraph of $G$.

Case $3\left|V\left(G_{1}\right)\right|=3 . G_{1}$ could be the graph in Figure 2.2.2 or 2.2 .3 since $G_{1}$ is connected and simple. Since $G_{1}$ has no 3-cycles, $G_{1}$ must be the graph in Figure 2.2.2. Then since $G$ has no essential edge cuts of size $1, v_{1}, v_{3} \in V(G)$. And using an argument similar to that in Case 2, we conclude that $G$ has a dominating Eulerian subgraph.

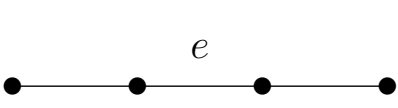

Figure 2.3.1

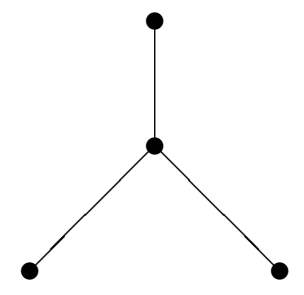

Figure 2.3.4

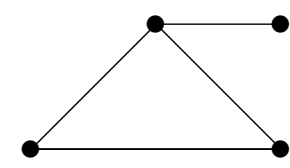

Figure 2.3.2

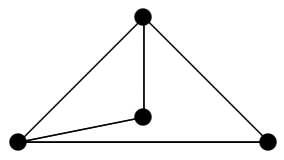

Figure 2.3.5

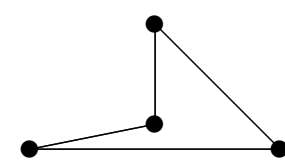

Figure 2.3.3

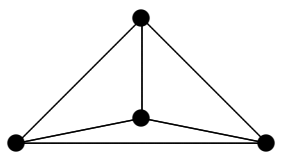

Figure 2.3.6

Case $4\left|V\left(G_{1}\right)\right|=4$ (see Figure 2.3).

Figure 2.3: $G_{1}$ 
In Figure 2.3.1, $G$ has an essential cut edge $e$, a contradiction; In Figure 2.3.3, by Lemma 2.2.2, $G$ has a spanning Eulerian subgraph. Since $G_{1}$ has no 3 -cycles, $G_{1}$ cannot be the graph illustrated in Figure 2.3.2, 2.3.5, 2.3.6. For Figure 2.3.4, we can use an argument similar to that used in Case 2 and 3 to conclude that $G$ has a dominating Eulerian subgraph.

A graph $G$ is $k$-triangular if each edge of $G$ is in at least $k$ triangles and $G$ is triangular if it is 1-triangular.

Lemma 2.2.4 Let $G$ be a simple connected graph that is not a path, a cycle or $K_{1,3}$, with $l(G)=l$. Then each of the following holds:

(i). For an integer $m, l \geq m \geq 0$,

$$
l\left(L^{m}(G)\right)=\left\{\begin{aligned}
l-m & : \quad \text { if } 0 \leq m<l \\
1 & : \quad \text { if } m \geq l
\end{aligned}\right.
$$

(ii). For integer $s \geq 0$,

$$
\delta\left(L^{l+s}(G)\right) \geq\left\{\begin{aligned}
2 & : \quad \text { if } s=0 \text { or } s=1 \\
2^{s-2}+2 & : \quad \text { if } s \geq 2
\end{aligned}\right.
$$

(iii). $L^{l}(G), L^{l+1}(G)$ and $L^{l+2}(G)$ are triangular and $L^{l+s}(G)$ is $2^{s-3}$-triangular when $s \geq 3$

(iv). For an integer $s \geq 0, \kappa\left(L^{l+s}(G)\right) \geq s+1$.

Proof. (i). Case $1 l(G)=1$.

By the definition of an arc, $l(G)=1$ if and only if one of the following holds
(A) $\delta(G) \geq 3$
(B) $\delta(G) \leq 2$ and every vertex of degree 2 is contained in a triangle. 
If (A) holds, then $\delta\left(L^{m}(G)\right) \geq 3$ and so $l\left(L^{m}(G)\right)=1$ for any $m \geq 0$. Hence, we assume that (B) holds. By way of contradiction we suppose that $l(L(G)) \geq 2$. Let $P_{0}=v_{0} v_{1} \cdots v_{l}$ be an arc of length of $l(L(G)) \geq 2$. So $d_{L(G)}\left(v_{1}\right)=d_{L(G)}\left(v_{2}\right)=\cdots=$ $d_{L(G)}\left(v_{1-1}\right)=2$. By the definition of an arc, $v_{0} v_{1} v_{2}$ is an induced path of length 2 in $L(G)$, i.e., $v_{0} v_{1}, v_{1} v_{2} \in E(L(G))$, but $v_{0} v_{2} \notin E(G)$. Assume that $e_{v_{0}}, e_{v_{1}}, e_{v_{2}}$ are edges in $G$ corresponding to $v_{0}, v_{1}, v_{2}$ in $L(G)$, respectively. So we have a path $e_{v_{0}}, e_{v_{1}}, e_{v_{2}}$ of length 3 in $G$ whose internal vertices are of degree 2 . Hence, $l(G) \geq 3$, contrary to $l(G)=1$.

Case $2 l(G) \geq 2$.

Let $P_{0}=v_{0} v_{1} \cdots v_{l}$ be an arc of length of $l(G) \geq 2$. By the definition of an arc, $d_{G}\left(v_{i}\right)=2$ for $i=1,2, \cdots, l-1$. Let $u_{1}, u_{2}, \cdots, u_{l}$ be the vertices in $L(G)$ corresponding to the edges $v_{0} v_{1}, v_{1} v_{2}, \cdots, v_{l-1} v_{l}$ in $G$ respectively. Then $u_{1} u_{2} \cdots u_{l}$ is an arc in $L(G)$ with length $l-1$. So $l(L(G))=l-1$. Inductively, we have $l\left(L^{m}(G)\right)=l-m$ when $0 \leq m<l$.

In particular, $l\left(L^{m}(G)\right)=1$ when $m \geq l$ by Case 1 since $l\left(L^{l-1}(G)\right)=1$.

(ii). First we prove that $\delta\left(L^{l}(G)\right) \geq 2$. We assume by way of contradiction that there exists a vertex $v$ of degree 1 in $L^{l}(G)$. So the edges corresponding to $v$ and its only adjacent vertex induce a path of length 2 with an internal vertex of degree 2 in $L^{l-1}(G)$, which is an arc of length 2, contrary to the fact that $l\left(L^{l-1}(G)\right)=1$. Hence, $\delta\left(L^{l}(G)\right) \geq 2$. For any graph $G$ that is neither a path nor a cycle, the sequence $\delta\left(L^{i}(G)\right), i=i, 2, \cdots$, is nondecreasing. So we also have $\delta\left(L^{l+1}(G)\right) \geq 2$.

Next we prove that $\delta\left(L^{l+2}(G)\right) \geq 3$.

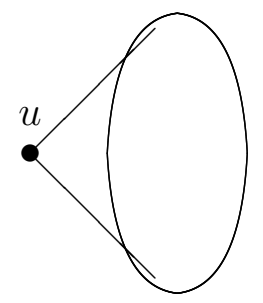

Figure 2.4.1: $L^{l+2}(G)$ 


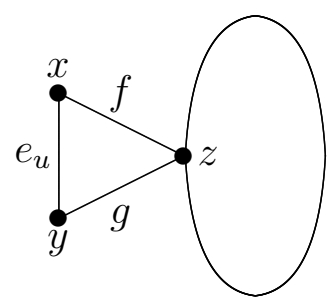

Figure 2.4.2: $L^{l+1}(G)$

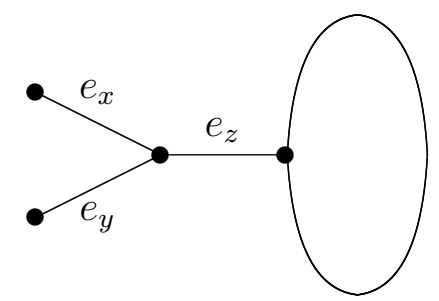

Figure 2.4.5: $L^{l}(G)$

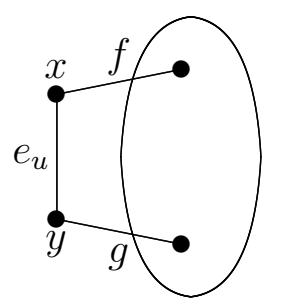

Figure 2.4.3: $L^{l+1}(G)$

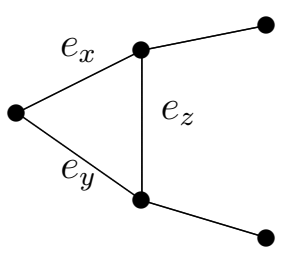

Figure 2.4.6: $L^{l}(G)$

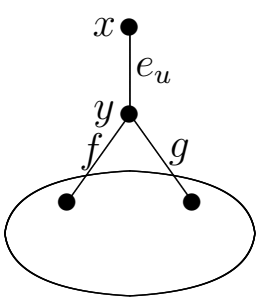

Figure 2.4.4: $L^{l+1}(G)$

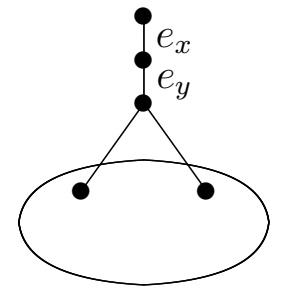

Figure 2.4.7: $L^{l}(G)$

Assume that there exists a vertex $u$ in $L^{l+2}(G)$ of degree 2 (see Figure 2.4.1), then the corresponding edge $e_{u}=x y$ of $u$ in $L^{l+1}(G)$ is incident with 2 edges. That means $e_{u}$ is either a pendent edge (see Figure 2.4.4) or an edge with both ends $x, y$ of degree 2 (see Figure 2.4.2 and 2.4.3). The graph $L^{l+1}(G)$ in Figure 2.4.4 corresponds to $L^{l}(G)$ in Figure 2.4.7, contrary to (i) that $l\left(L^{l}\right)(G)=1$. So we can assume that the end vertices of $e_{u}$ both have degree 2. Then $E_{L^{l+1}(G)}(x)=\left\{e_{u}, f\right\}$ and $E_{L^{l+1}(G)}(y)=\left\{e_{u}, g\right\}$. And it is easy to see that $\left\{f, e_{u}, g\right\}$ forms an arc of length 3 if $f$ and $g$ have no common vertices (see Figure 2.4.3), contrary to the fact that $L^{l+1}(G)$ has no arcs of length greater than 1 by (i). So $\left\{f, e_{u}, g\right\}$ forms a triangle in $L^{l+1}(G)$ (see Figure 2.4.2). Assume that $e_{x}, e_{y}, e_{z}$ are edges in $L^{l}(G)$ corresponding to the vertices $x, y, z$ in $L^{l+1}(G)$. The graph induced by $\{x, y, z\}$ corresponds to a $K_{1,3}$ (see Figure 2.4.5) or $C_{3}$ (see Figure 2.4.6). First consider the case of Figure 2.4.6. Since $G$ is not a $C_{3}$ nor a $K_{1,3}, L^{l}(G)$ can not be a $C_{3}$. So $e_{z}$ is adjacent to at least one more edge other than $e_{x}, e_{y}$, which is contrary to the fact that $e_{x}$ and $e_{y}$ can only be adjacent to $e_{z}$. Since $l \geq 1, L^{l}(G)$ is a line graph and so it is claw free, which excludes the graph in Figure 2.4.5.

So we have $\delta\left(L^{l+2}(G)\right) \geq 3$.

Define $a_{1}=3, a_{2}=4$. Since $\delta\left(L^{l+2}(G)\right) \geq 3=a_{1}$, every edge in $L^{l+2}(G)$ is adjacent to at least $4=2(3-1)=a_{2}$ edges and so $\delta\left(L^{l+3}(G)\right) \geq 4=a_{2}$. Inductively, suppose 
that every edge in $L^{l+s-1}(G)$ is adjacent to at least $a_{s-1}=2 a_{s-2}-2$ edges. Then $\delta\left(L^{l+s}(G)\right) \geq 2^{s-2}+2$, as desired.

(iii). First we prove that $L^{l}(G)$ is triangular; that is, for any $e=x y \in E\left(L^{l}(G)\right)$, e lies in at least one triangle. If $e$ is a pendent edge in $L^{l}(G)$ (see Figure 2.5.2), then $\delta\left(L^{l}(G)\right)=1<2$, contrary to (ii). And since $l\left(L^{l}(G)\right)=1$, if one of $d_{L^{l}(G)}(x)$ and $d_{L^{l}(G)}(y)$ is 2 , then $e$ must lie in a triangle. So we can assume that $d_{L^{l}(G)}(x) \geq 3$ and $d_{L^{l}(G)}(y) \geq 3$ (see Figure 2.5.1). Since $x y \in E\left(L^{l}(G)\right.$ ), the corresponding edges $e_{x}$ and $e_{y}$ in $L^{l-1}(G)$ share a common vertex $v$ in $L^{l-1}(G)$. So the corresponding $L^{l-1}(G)$ of $L^{l}(G)$ is a graph in Figure 2.5.3 or Figure 2.5.4 by $l\left(L^{l-1}(G)\right)=1$. Then $e$ lies in a triangle in $L^{l}(G)$.

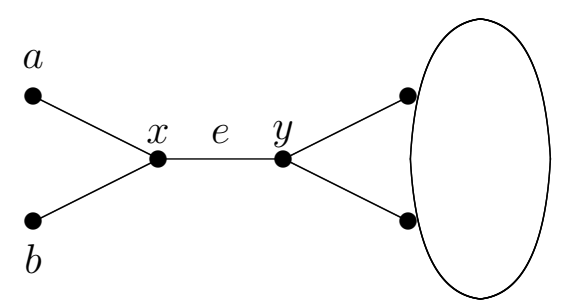

Figure 2.5.1: ${ }_{v^{\prime}}^{L}(G)$

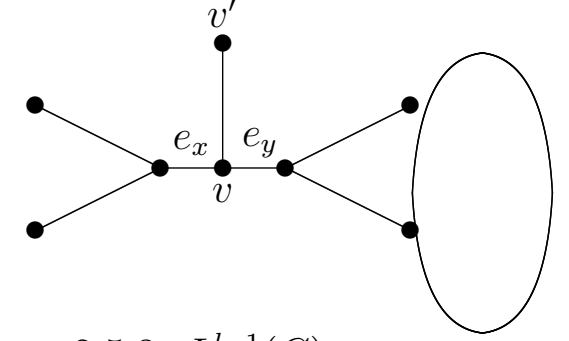

Figure 2.5.3: $L^{l-1}(G)$

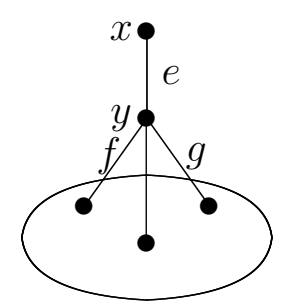

Figure 2.5.2: $L^{l}(G)$

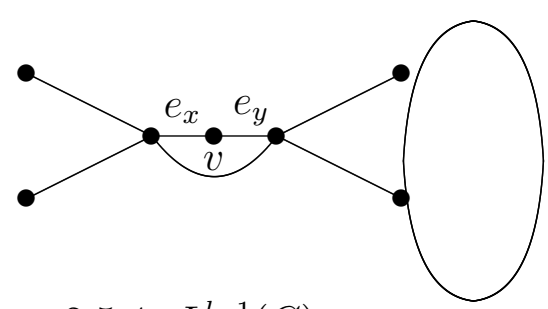

Figure 2.5.4: $L^{l-1}(G)$

Figure 2.5

Let $\left.x y \in E\left(L^{l+1}\right)\right)$. Then $e_{x}$ and $e_{y}$ share a common vertex in $L^{l}(G)$. Since $e_{x}$ lies in a triangle, there exists an edge $e_{a}$ that is incident with both $e_{x}$ and $e_{y}$. Hence, $L^{l+1}(G)$ is triangular. Similarly, $L^{l+2}(G)$ is triangular. And so $L^{l+1}(G)$ and $L^{l+2}(G)$ are both triangular.

If $s \geq 3$, by (ii), $\delta\left(L^{l+s-1}(G)\right) \geq 2^{s-3}+2$, so the incident edges of each vertex form a 
complete graph with order at least $2^{s-3}+2$ in $L^{l+s}(G)$. Then each edge of $L^{l+s}(G)$ lies in at least $2^{s-3}$ triangles, that is, $L^{l+s}(G)$ is $2^{s-3}$-triangular since $L^{l+s}(G)$ is the edge-disjoint union of complete graphs.

(iv) Since $G$ is connected, $L^{l}(G)$ is connected, i.e., $\kappa\left(L^{l}(G)\right) \geq 1$. So we now assume $s \geq 1$.

Notice that, for an integer $k \geq 0$, a non-complete line graph $L(H)$ has no vertex cut of size less than $k$ if and only if $H$ has no essential edge cut of size less than $k$. Next we prove that $\kappa\left(L^{l+s}(G)\right) \geq s+1$ by showing that $L^{l+s-1}(G)$ has no essential edge cut of size less than $s+1$. Suppose that $L^{l+s-1}(G)$ has an essential edge cut $X$ of size less than $s+1$ and $L^{l+s-1}(G)-X$ has two nontrivial components $C_{1}$ and $C_{2}$. Since $L^{l+k}(G)$ is connected and triangular, $|X| \geq 2$.

Since $|X| \geq 2, L^{l}(G)$ has no essential edge cuts of size 1 , and so $\kappa\left(L^{l+1}(G)\right) \geq 2$.

If $k=2$ and $|X|=2$, then there must exist a vertex $v$ in $C_{1}$ or $C_{2}$ such that $X \subseteq E_{L^{l+1}(G)}(v)$. Since $X$ is essential, $v$ is incident with one more edge except $X$. Then $v$ is a cut vertex of $L^{l+1}(G)$, a contradiction. So $|X| \geq 3$, that is, $\kappa\left(L^{l+2}(G)\right) \geq 3$.

Next we prove that $\kappa\left(L^{l+s}(G)\right) \geq s+1$ when $s \geq 3$ by induction. Assume that $\kappa\left(L^{l+s-1}(G)\right) \geq s$ and we consider the graph $L^{l+s-1}(G)$. Since each edge lies in at least one triangle, at least two edges of $X$ are incident with the same vertex. So we can assume without loss of generality that $X$ has at most $s-1$ vertices in $C_{1}$ and denote the set of these vertices by $Y$. Since $|X| \leq s$, at least one vertex $y$ of $Y$ is incident with exactly one edge of $X$. Since $\delta\left(L^{l+s-1}(G)\right) \geq 2^{s-3}+2 \geq s$ when $s \geq 3, d_{L^{l+s-1}(G)}(v) \leq s-2+1=s-1$, a contradiction. Hence $E\left(C_{1}-Y\right) \neq \emptyset$. So $Y$ is a $(s-1)$-cut of $L^{l+s-1}(G)$, contrary to the induction hypothesis.

Therefore $L^{l+s}(G)$ has no essential edge cuts of size less than $s+2$ and so $\kappa\left(L^{l+1+s}(G)\right) \geq$ $s+2$.

Lemma 2.2.5 Let $G$ be a simple connected graph that is neither a path nor a cycle with $l(G)=l$. Then for any $S^{\prime} \subseteq E\left(L^{l+s}(G)\right)$ with $\left|S^{\prime}\right| \leq s, L^{l+s}(G)-S^{\prime}$ has a dominating Eulerian subgraph. 
Proof By Lemma 2.2.4(iii), $L^{l+s}(G)$ is $\left(2^{s-3} \geq 8 \geq s+1\right)$-triangular when $s \geq 6$. In this case, every edge of $L^{l+s}(G)-S^{\prime}$ lies in at least one triangle since $\left|S^{\prime}\right|=s$. By Lemma 2.2.3, $L^{l+s}(G)-S^{\prime}$ has a spanning Eulerian trail since we can contract all the triangles to get a $K_{1}$. Next we consider the cases when $s=0,1,2,3,4,5$.

Since $L^{l+s}(G)$ is an edge-disjoint union of complete graphs, we can assume that $\left\{E_{1}, E_{2}, \cdots, E_{n}\right\}$ is a complete edge partition of $L^{l+s}(G)$ where $E_{i}$ is a complete graph for $1 \leq i \leq n$. Consider $L^{l+s}(G)-S^{\prime}$ and notice that deleting some edges in $L^{l+s}(G)$ may result in some of the edges in $L^{l+s}(G)-S^{\prime}$ not lying in any triangles. For any complete $E_{i}$ with order $t$, if $E_{i} \cap S^{\prime}=\emptyset$, then $E_{i}$ is still triangular; if $E_{i} \cap S^{\prime} \neq \emptyset$ and $E_{i}-S^{\prime}$ is not triangular, then

$$
\left|E_{i} \cap S^{\prime}\right| \geq t-2 .
$$

Let $G_{1}$ be the graph obtained by contracting all the triangles and multiple edges repeatedly from $L^{l+s}(G)-S^{\prime}$. From the proof of Lemma 2.2.4 (iv), $L^{l+s}(G)$ has no essential edge cuts of size less than $s+2$. Hence $L^{l+s}(G)-S^{\prime}$ has no essential edge cuts of size less than 2. By Lemma 2.2.3, it suffices to show that $\left|V\left(G_{1}\right)\right| \leq 4$ in each of the cases below, we shall show that $|V(G)| \leq 4$.

Case $1 s=0$. Since $L^{l}(G)$ is triangular, then $G_{1}=K_{1}$.

Case $2 s=1$. Let $S^{\prime}=\{e\}$ and consider $L^{l+1}(G)-S^{\prime}$. By (1), the only possibility of making some $K_{t}-S^{\prime}$ not triangular is that $S^{\prime} \subseteq K_{3}$. We can assume that $E_{1}=K_{3}$ and by (1), for any $e \in E_{2} \cup E_{3} \cdots \cup E_{n}$, e lies in at least one triangle of $L^{l+1}(G)-S^{\prime}$. That means the induced graph of $E_{2} \cup E_{3} \cdots \cup E_{n}$ is triangular. Since $\kappa\left(L^{l+1}(G)\right) \geq 1+1=2$, $E_{1}=K_{3}$ shares at least two vertices with other $E_{i}$ 's. Thus $G_{1}$ has at most 2 vertices left.

Case $3 s=2$. Let $S^{\prime}=\left\{e_{1}, e_{2}\right\}$ and consider $L^{l+2}(G)-S^{\prime}$. By (1), the possibilities of making some $K_{t}-S^{\prime}$ not triangular are listed below (see Table 1).

Table 1 


\begin{tabular}{|c|c|c|c|c|}
\hline & $e_{1}$ & $e_{2}$ & $\left|V\left(G_{1}\right)\right| \leq$ & $\left|E\left(G_{1}\right)\right| \leq$ \\
\hline Case 2.1 & $K_{4}$ & $K_{4}$ & 4 & 2 \\
\hline Case 2.2 & $K_{3}$ & $K_{3}$ & 3 & 1 \\
\hline Case 2.3 & $K_{3}$ & $K_{3}^{\prime}$ & 5 & 4 \\
\hline
\end{tabular}

In Table 1, Case 2.1 is the case when $e_{1}, e_{2}$ are contained in the same $K_{4}$; Case 2.2 is the case when they are both contained in the same $K_{3}$; Case 2.3 is the case when one of them is contained in a $K_{3}$ and the other is contained in a different $K_{3}$.

Let $X$ be the union of the complete graphs each of which contains some edges of $S^{\prime}$. So we have that $X=K_{3}$ (Case 2.2), $X=K_{4}$ (Case 2.1) or $X=K_{3} \cup K_{3}^{\prime}$ (Case 2.3) and for any $e \in G-X$, $e$ lies in at least one triangle of $L^{l+2}(G)-S^{\prime}$. Since $\kappa\left(L^{l+2}(G)\right) \geq 2+1=3$, $X$ shares at least three vertices with other $E_{i}$ 's.

If $X=K_{3}$, then $G_{1}$ has at most 1 vertex left; if $X=K_{4}$, then $K_{4}$ has at most two vertices left; if $X=K_{3} \cup K_{3}^{\prime}$, then $G_{1}$ has at most 4 vertices left.

Case $4 s=3$. Let $S^{\prime}=\left\{e_{1}, e_{2}, e_{3}\right\}$ and consider $L^{l+3}(G)-S^{\prime}$. By Lemma 2.2.4(ii), $\delta\left(L^{l+2}(G)\right) \geq 3$. So each $E_{i}$ is an edge-disjoint union of complete graphs with orders at least 3. By (1), the possibilities of making some $K_{n}-S^{\prime}$ not triangular are that either $S^{\prime}$ is contained in some $K_{5}$, or at least 2 edges of $S^{\prime}$ are contained in some $K_{4}$, or at least 1 edge of $S^{\prime}$ is contained in some $K_{3}$.

Suppose that at least 2 edges of $S^{\prime}$ are contained in some $K_{4}$, or at least 1 edge of $S^{\prime}$ is contained in some $K_{3}$. Then since edges of $L^{l+3}(G)$ not in these complete graphs are lying in triangles disjoint from these complete graphs, and since $\kappa\left(L^{l+3}(G)\right) \geq 3+1=4$, the contraction of all the triangles of $L^{l+s}(G)-S^{\prime}$ will result in a graph $G_{1}$ with at most 4 vertices.

Now suppose that a $K_{5}$ contains all 3 edges in $S^{\prime}$. Since $\kappa\left(L^{l+3}(G)\right) \geq 3+1=4$, this $K_{5}$ shares at least four vertices with other $E_{i}$ 's. And so $G_{1}$ has at most 2 vertices left.

Case $5 s=4$. Let $S^{\prime}=\left\{e_{1}, e_{2}, e_{3}, e_{4}\right\}$ and consider $L^{l+4}(G)-S^{\prime}$. By Lemma 2.2.4(ii), $\delta\left(L^{l+3}(G)\right) \geq 4$. So each $E_{i}$ is an edge-disjoint union of complete graphs with orders at least 4. By (1), the possibilities of making some $K_{n}-S^{\prime}$ not triangular are that either $S^{\prime}$ 
is contained in some $K_{6}$, or at least 3 edges of $S^{\prime}$ are contained in some $K_{5}$, or at least 2 edges of $S^{\prime}$ are contained in some $K_{4}$.

Suppose that at least 3 edges of $S^{\prime}$ are contained in some $K_{5}$, or at least 2 edge of $S^{\prime}$ is contained in some $K_{4}$. Then since edges of $L^{l+4}(G)$ not in these complete graphs are lying in triangles disjoint from these complete graphs, and since $\kappa\left(L^{l+4}(G)\right) \geq 5$, the contraction of all the triangles of $L^{l+s}(G)-S^{\prime}$ will result in a graph $G_{1}$ with at most 4 vertices.

Now suppose that a $K_{6}$ contains all 4 edges in $S^{\prime}$. Since $\kappa\left(L^{l+4}(G)\right) \geq 4+1=5$, this $K_{6}$ shares at least five vertices with other $E_{i}$ 's. And so $G_{1}$ has at most 2 vertices left.

Case $6 s=5$. Let $S^{\prime}=\left\{e_{1}, e_{2}, e_{3}, e_{4}, e_{5}\right\}$ and consider $L^{l+5}(G)-S^{\prime}$. By Lemma 2.2.4(ii), $\delta\left(L^{l+3}(G)\right) \geq 6$. So each $E_{i}$ is an edge-disjoint union of complete graphs with orders at least 6. By (1), the possibilities of making some $K_{n}-S^{\prime}$ not triangular are that either $S^{\prime}$ is contained in some $K_{7}$ or 4 edges of $S^{\prime}$ are contained in some $K_{6}$. Since $\kappa\left(L^{l+5}(G)\right) \geq 5+1=6$, this concerned $K_{7}$ or $K_{6}$ shares at least six vertices with other $E_{i}$ 's. And so $G_{1}$ has at most 2 vertices left.

Proof of Theorem 2.1.1 Let $S$ be a vertex set of $L^{l+1+s}(G)$ with $|S|=s$. Let $S^{\prime}$ be the edge set of $L^{l+s}(G)$ corresponding to $S$ in $L^{l+1+s}(G)$. By Lemma 2.2.4(iv), $\kappa\left(L^{l+1+s}(G)\right) \geq$ $s+2$. Then $\kappa\left(L^{l+1+s}(G)-S\right) \geq 2$ and so $L^{l+s}(G)-S^{\prime}$ has no essential edge cuts of size 1 , or equivalently, all the edge cuts of size 1 of $L^{l+s}(G)-S^{\prime}$ are pendent edges. And since $\kappa^{\prime}\left(L^{l+s}(G)\right) \geq \kappa\left(L^{l+s}(G)\right) \geq s+1$ and $\left|S^{\prime}\right|=s, \kappa^{\prime}\left(L^{l+s}(G)-S^{\prime}\right) \geq 1$. So $L^{l+s}(G)-S^{\prime}$ is connected. By Lemma 2.2.5, $L^{l+s}(G)-S^{\prime}$ has a dominating Eulerian subgraph. Since deleting an edge in $L^{l+s}(G)$ will not affect the adjacency relationship between any two edges in $E\left(L^{l+s}(G)\right)-S^{\prime}$, the adjacency relationship between any two corresponding vertices in $V\left(L^{l+s+1}(G)\right)-S$. So the induced graph of $V\left(L^{l+s+1}(G)\right)-S$ in $L^{l+s+1}(G)$ is the line graph of the induced graph of $E\left(L^{l+s}(G)\right)-S^{\prime}$ in $L^{l+s}(G)$. Then by Theorem 2.2.1, $L^{l+1+s}(G)-S$ is hamiltonian. And so $L^{l+1+s}(G)$ is s-hamiltonian.

From Theorem 2.1.1, we know the $s$-Hamilton index of a graph $G h_{s}(G)$ is at most $l+s+1$. It is natural to ask what kind of graphs have $h_{s}(G)=l+s+1$.

Question 1 Characterize the graph $G$ with $h_{s}(G)=l+s+1$. 
Question 2 If $h_{s}(G)<l+s+1$, is the graph $L^{h_{s}+k}(G)$ is also $s$-hamiltonian for any integer $k \geq 1$ ?

Question 3 Is this line graph model an optimal model for $s$-fault-tolerant network? 


\section{Bibliography}

[1] J. A. Bondy and U. S. R. Murty, Graph theory with applications, Macmillan, London and Elsevier, New York, 1976.

[2] G. Chartrand, C. E. Wall, On the hamiltonian index of a graph, Studia, Sci. Math. Hungar. 8 (1973), 43-48.

[3] L. H. Clark and N. C. Wormald, Hamilton-like indices of graphs, Ars Combinatoria 15 (1983), 131-148.

[4] F. Harary and C. St. J. A. Nash-Williams, On Eulerian and Hamiltonian graphs and line graphs, Canad. Math. Bull. 8 (1965), 701-709.

[5] H.-J. Lai, On the hamiltoinan index, Discrete Math. 69 (1988), 43-53.

[6] L. Lesniak-Foster, On n-Hamilton Line graphs, J. Combin. Theory Ser. B, 22 (1977), 263-273. 